\title{
Call for Special Issue Papers: The Social Impact of Deep Fakes
}

\author{
Deadline for Manuscript Submission: March 1, 2020
}

\author{
Guest Editors: Jeremy Bailenson, PhD and Jeff Hancock, PhD; Stanford University
}

The era of deep fakes is upon us. Images, audio, videos, and textual information can easily be transformed or generated from scratch with Al technology. While synthetic media has been possible for decades, due to advances in machine learning and computer vision, now the transformations can occur in real-time. Also, the technology has migrated from specialized labs to apps anyone can buy for a few dollars.

There has been ample work done in computer science on automatic generation and detection of deep fakes, but to date there have only been a handful of social scientists who have examined the social impact of deep fakes. Most of this initial work concerns the ability of humans to detect them. It is time to understand any possible effects deep fakes might have on people, and how psychological and media theories apply to this new technology.

We are seeking original articles on the following topics related to the psychological dynamics around deep fakes. Research topics might include:

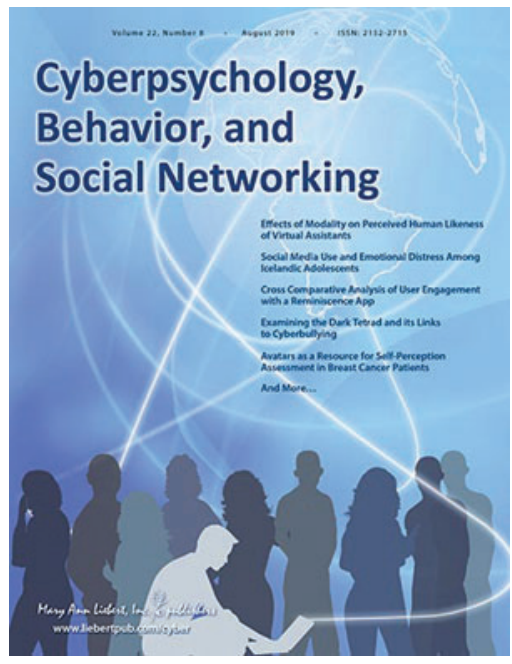

- The impact of receiving altered versions of oneself and/or others

- How exposure to deep fakes undermines trust in media

- Possible therapeutic interventions based on deep fakes

- How technological immersion interacts with deep fakes

- How enduring and resilient deep fakes can be

- Are strategies around debunking or countering deep fakes effective?

- Understanding the use of deep fakes in self-presentation during social interaction

Access the Instructions for Authors before submitting your manuscript. Visit the Cyberpsychology, Behavior, and Social Networking website (www.liebertpub.com/cyber) to learn more and read past issues.

\section{Questions?}

Contact the Journal Editorial Office at cyberpsych@vrphobia.com.

"Submitting Authors: Please indicate in your cover letter that your paper is for the "Deep Fakes" Special Issue, and upload your paper under the "Deep Fakes" manuscript category.

\section{Submit your paper for peer review online:} https://mc.manuscriptcentral.com/cyber 